\title{
Degeneration of axons in the corticospinal tract secondary to spinal cord ischemia in rats
}

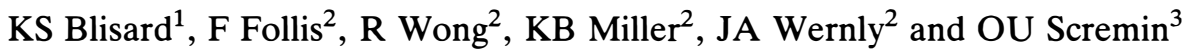 \\ ${ }^{1}$ Department of Pathology \& Laboratory Medicine, University of Cincinnati Medical Center, Cincinnati, OH \\ 45267-0529; ${ }^{2}$ Department of Thoracic \& Cardiovascular Surgery, University of New Mexico, Albuquerque, NM \\ 87108; ${ }^{3}$ West Los Angeles VA Medical Center and Department of Physiology at the University of California Los \\ Angeles School of Medicine, Los Angeles, CA 90073, USA
}

\begin{abstract}
Occlusion of the thoracic aorta and both subclavian arteries (XC) in the rat model produces spastic paraplegia. In order to characterize the lesion of white matter, 14 male Sprague-Dawley rats underwent XC for 10.5 to $12 \mathrm{~min}$, were observed for 32 days and assessed with a lesion score. A sham group of eight underwent surgical manipulations without XC. The spinal cords were studied by optical microscopy and electron microscopy. An additional group of normal animals $(n=8)$ underwent spinal cord blood flow measurement with the autoradiographic technique. Optical microscopy showed normal histology in sham operated rats and rats with aortic cross-clamp and lesion score $=2-4$ $(n=5)$, rare changes in the white matter of rats with lesion score $=8(n=2)$, and demyelination of the anterior and lateral tracts of the white matter and motor neuron loss in the gray matter of rats with lesion score $=13-15(n=7)$ and spastic paraplegia. In this last group, electron microscopy disclosed severe axonal degeneration of corticospinal tracts. In the same region spinal cord blood flow was higher than the remaining white matter. This study confirms that spastic paraplegia observed in the rat model after XC is due to degeneration of the pyramidal tracts, perhaps more susceptible to injury due to the high spinal cord blood flow.
\end{abstract}

Keywords: aortic occlusion; paraplegia; blood flow; corticospinal tract axon degeneration; spinal cord ischemia

\section{Introduction}

Spinal cord ischemia associated with aortic surgery occasionally can result in the devastating complication of paraplegia. Recently, ischemic paraplegia has been described, in the rat model, by LeMay et $a l^{1}$ after occlusion of the descending thoracic aorta and both subclavian arteries for various periods of time. A previous investigation by our group ${ }^{2}$ with this model has shown that the paraplegia is characterized by spasticity and that the injury is localized, as seen on histology, to the white matter of the lateral and ventral portions of the spinal cord, as well as, in some animals, to the ventral portion of the gray matter. The posterior columns appeared intact on light microscopy. In addition, spinal cord conduction time (SCCT), obtained from the difference of sensory evoked potentials from stimulation of the hindpaw and forepaw, was increased.

In the rat, the corticospinal tracts course in the deepest portion of the posterior columns, just above

Correspondence: Fabrizio Follis, MD, University of New Mexico, Department of Thoracic \& Cardiovascular Surgery, 2211 Lomas Blvd, Albuquerque, NM 87131, USA the central gray (Figure 1), while afferents related to the short latency sensory evoked potentials are believed to travel primarily through the posterior columns. Since we could not resolve the paradox of a clinical presentation of spastic paraplegia with intact corticospinal tracts, and of increased spinal cord conduction time with undamaged posterior columns on the basis of the light microscopy, we designed the present study to detect the changes at an ultrastructural level, if any, of the descending and ascending tracts of the posterior columns. In addition, in order to determine the possible role of hemodynamic factors in the selective vulnerability of corticospinal fibers, blood flow in the region of the corticospinal tract was evaluated with the iodo- ${ }^{14} \mathrm{C}$-antipyrine autoradiographic technique, in rats subjected to median sternotomy without vascular occlusion.

\section{Methods}

Light and electron microscopy study

Fourteen male Sprague-Dawley rats underwent occlusion of the descending thoracic aorta and both subclavian arteries (XC) for periods of 10.5, 11 and $12 \mathrm{~min}$ 


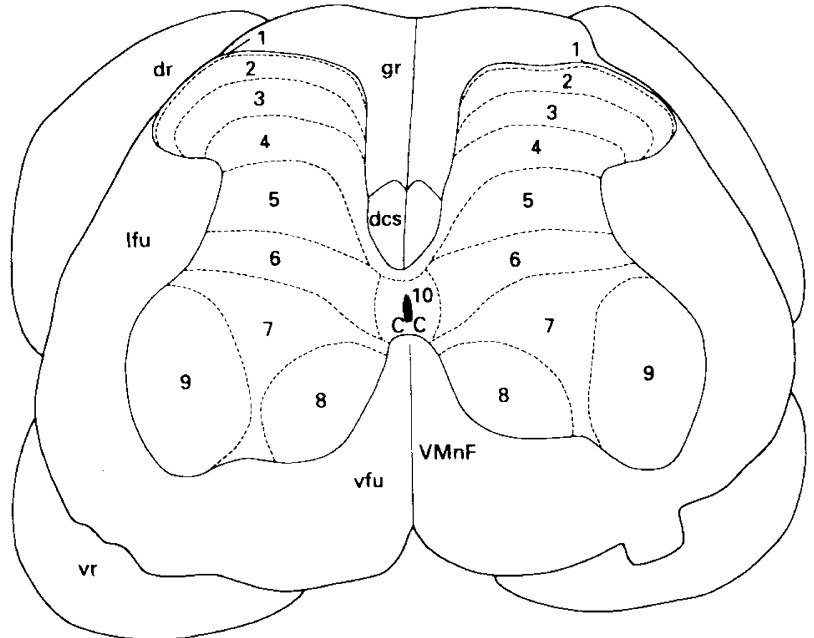

Figure 1 Outline of rat spinal cord at L5. Central canal (CC), dorsal corticospinal tract (dcs), dorsal root of spinal nerve (dr), gracile fasciculus (gr), lateral funiculus of spinal cord (lfu), ventral funiculus of spinal cord (vfu), ventral median fissure of spinal cord (VMnF), ventral root of spinal nerve (vr). The ten layers of Rexed in the spinal cord are indicated by the numerals $1-10$. (Reprinted with permission)

according to the methods previously described. ${ }^{1,2}$ An additional group of eight underwent the surgical procedures without XC (sham). All the animals were kept alive for 32 days. At the end of this period the neurological function was appraised with a semiquantitative numerical score $(0-15)$ described by LeMay et $a l^{1}$ where 15 represented a paraplegic animal and zero a normal one (Table 1). Then the animals were euthanized with an overdose of halothane and perfused in situ with a modified Karnovsky's solution. Cross sections were taken from the spinal cord in the cervical, thoracic and lumbar regions for paraffin processing. Sections were cut at $5 \mu \mathrm{m}$ and stained with hematoxylin and eosin and with Luxol fast blue with periodic acidSchiff's reagent. In addition, cross sections taken from the lumbar cord were post-fixed in osmium tetroxide and processed into Spurr's resin. Semi-thin sections were cut at $1 \mu \mathrm{m}$ and stained with methylene blue-azure II-basic fuchsin and examined by light microscopy. Finally, thin sections were cut at $100 \mathrm{~nm}$ from selected blocks, stained with uranyl acetate and lead citrate and examined with an Hitachi electron microscope.

\section{Spinal cord blood flow (SCBF) study}

Under endotracheal halothane anesthesia, eight male Sprague-Dawley rats underwent median sternotomy, dissection of the vessels and placements of tourniquets without occlusion. Then the spinal cord blood flow was measured with the tracer washout technique employing the iodo- ${ }^{14} \mathrm{C}$-antipyrine (IAP) method as described by Sakurada et al. ${ }^{3}$ Two arterial and two venous catheters were implanted in the femoral vessels. One arterial catheter was connected to a pressure transducer for
Table 1 Lesion scoring system for neurological function (after LeMay et al)

Deficit score

Walking with lower extremities ( $L E)$

0 No evidence of deficit

1 Toes flat under body when walking but ataxia exists

2 Knuckle walks

3 Movements in LE but no knuckle walk

4 No movements, drags LE

Horizontal rope platform ${ }^{\text {a }}$

0 Grasps rope and pulls up with LE

1 Raises LE and grasps rope without pulling

2 Raises LE but cannot grasp rope

3 Does not raise LE

Rotating screen ${ }^{\mathrm{b}}$

0 LE grasp screen to 180 degrees $>5 \mathrm{~s}$

1 LE grasp screen to 180 degrees $<5 \mathrm{~s}$

2 LE grasp screen past vertical but not to 180 degrees

3 LE fall from screen past vertical (270-180 degrees)

Wooden bar 1 in in diameter at 45 degrees $^{c}$

0 LE grasp bar $>10 \mathrm{~s}$

1 LE grasp bar $>5 \mathrm{~s}$

2 LE grasp bar $<5 \mathrm{~s}$

3 LE slide off bar without grasping

Pain sensation

0 Withdrawal to toe pinch

1 Reacts or squeals to toe pinch but does not withdraw 2 No reaction to toe pinch

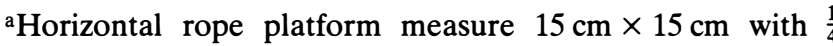
inch nylon rope; the upper extemities are placed on platform edge and a normal rat pulls LE up onto platform ${ }^{b}$ A square wire screen, $30 \mathrm{~cm} \times 30 \mathrm{~cm}$ in size, with $\frac{1}{4}$ inch holes; the rat is placed on horizontal screen, ( 0 degree $)$ then the screen is rotated down through the vertical position (270 degrees) to the inverted position (180 degrees). A normal rat grasps the screen with $L E$ for $>5 \mathrm{~s}$ when rotated to 180 degrees

'Wooden bar is $50 \mathrm{~cm}$ long and $2.5 \mathrm{~cm}$ wide; a normal rat grasps the wooden bar with LE for more than $10 \mathrm{~s}$

Printed with permission from D R Lemay et al 'Paraplegia in the rat induced by aortic cross-clamping: Model characterization and glucose exacerbation of neurologic deficit', J Vasc Surg 1987; 6: 384.

continuous recording of blood pressure, the other was used to sample arterial blood during IAP infusion. One venous catheter was used for IAP infusion, the other for infusion of the euthanasia agent. A $0.3 \mathrm{ml}$ sample of blood was obtained to measure $\mathrm{PaCO}_{2}, \mathrm{PaO}_{2}$ and $\mathrm{pH}$ in a Radiometer ABL 30 blood gas machine. Immediately after, an infusion of ${ }^{14} \mathrm{C}$-IAP was started. Infusate volume was $0.6 \mathrm{ml}$, dose $100-125 \mu \mathrm{Ci} / \mathrm{kg}$ body weight and infusion period $30 \mathrm{~s}$. Arterial blood samples $(20 \mu \mathrm{l})$ were obtained every $3 \mathrm{~s}$ from a free flowing arterial catheter until cardiac arrest occurred and shortly thereafter. At the end of IAP infusion, circulation was arrested by an i.v. pulse of euthanasia solution of $0.8 \mathrm{ml}$ of $3 \mathrm{MKCI}$. The exact time of cardiac arrest was determined from a continuous record of arterial blood 
pressure obtained on a polygraph; the samples obtained after cardiac arrest were discarded, the longest possible interval between sampling and cardiac arrest was $2 \mathrm{~s}$. Following this, samples (approximately $1 \mathrm{~cm}$ ) of cervical, thoracic and the entire lumbosacral cord were removed and flash frozen in methylbutane chilled to $-70^{\circ} \mathrm{C}$. They were then transferred to a cryostat and embedded in OCT compound for later sectioning.

The various segments of spinal cord to be studied were sectioned in a cryostat in $20 \mu \mathrm{m}$ thick slices, at $400 \mu \mathrm{m}$ intervals, then mounted on glass slides and heat dried on a hot plate at $60^{\circ} \mathrm{C}$ for at least $5 \mathrm{~min}$. These dried sections were then exposed to Kodak NMC film for 4 weeks, along with eight poly-methyl-methacrylate ${ }^{14} \mathrm{C}$ standards of known radioactivity. After exposure, the films were developed on a Kodak XO-Mat processor. Optical density induced in the film by the slides and standards was quantified with a scanning microscope densitometer. Regional spinal cord blood flow (rBF) was calculated form the values of blood radioactivity in the samples collected during the last $30 \mathrm{~s}$ of the experiment (during IAP infusion). IAP concentration as a function of time was introduced in the operational equation described by Reivich et al $^{4}$ that, when solved for a series of incremental values of $\mathrm{rBF}$, yielded a table relating blood flow to tissue radioactivity. This table permitted the calculation of $\mathrm{rBF}$ from the microdensitometric data obtained from the autoradiographs.

Five white matter regions, medial dorsal column (MDC), ventral column (VC), lateral column (LC), lateral dorsal column (LDC), corticospinal tract (CST) and one gray matter region, ventral horn (VH1) were examined in the cervical, thoracic and lumbar segments of the spinal cord.

\section{Results}

\section{Light and electron microscopy study}

Untreated or sham-operated animals $(n=8)$ showed normal spinal cord morphology on light microscopy in both paraffin- and resin-embedded sections (Figre $2 a$ ). By electron microscopy, normal axonal structure and distribution were observed in the area of the corticospinal tracts (Figre 3a).

Treated rats were divided into three groups based on the lesion scores. Animals with low lesion scores (LS $=2-4, n=5$ ) showed essentially normal morphology by light and electron microscopy. Axonal structure, myelination and fiber distribtution were unremarkable.

Two animals had moderate lesion scores ( $\mathrm{LS}=8)$. Rare PAS-positive macrophages were seen in the white matter in paraffin sections and rare degenerating axons were seen in resin-embedded material from these rats. No involvement of the gray matter was present.

Seven rats had high lesion scores (LS $=13-15)$ and spastic paraplegia. In paraffin sections from all these animals, severe degeneration was seen in the gray matter of the spinal cord, predominantly in the ventral horns. The appearance of the degeneration was similar
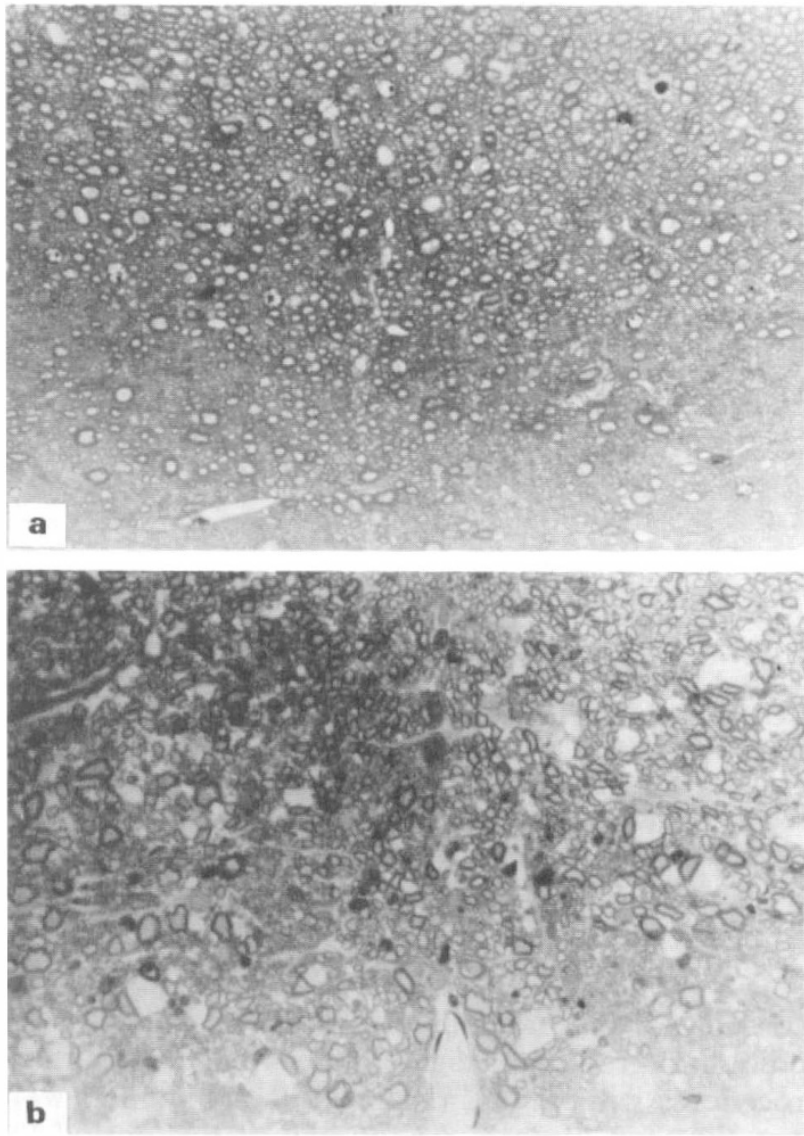

Figure 2 Semi-thin sections of lumbar cord. (a) Control rat has normal distribution of small and large fibers as well as normal fiber morphology. (b) Cord from spastic paraplegic rat demonstrates swelling and degeneration of fibers. Methylene blue-azure II-basic fuchsin on resin-embedded sections, $\times 250$

to that which was described in the previous report. Briefly, the gray matter was shrunken and collapsed with gliosis, accumulation of PAS-positive macrophages and loss of motor neurons. In the white matter, there were variable numbers of PAS-positive macrophages in the lateral and ventral columns. Again no change in the dorsal columns could be seen by light microscopy on paraffin sections. The structure of paraffin-embedded myelinated nervous tissue is difficult to interpret. Thus, we examined similar sections which had been processed into Spurr's resin. This technique is advantageous because the myelin, with its high concentration of lipids, is better preserved after osmium post-fixation, and the structure and distribution of myelinated fibers can be more accurately evaluated.

In resin-embedded sections from these animals, degenerative changes could be seen in the ventral-most portion of the dorsal columns just above the gray matter (Figure 2b). Some animals showed edema and separation of the fibers. In other animals, the axons were noticeably swollen or degenerated. In most rats, these changes appeared to be limited to a very discrete 

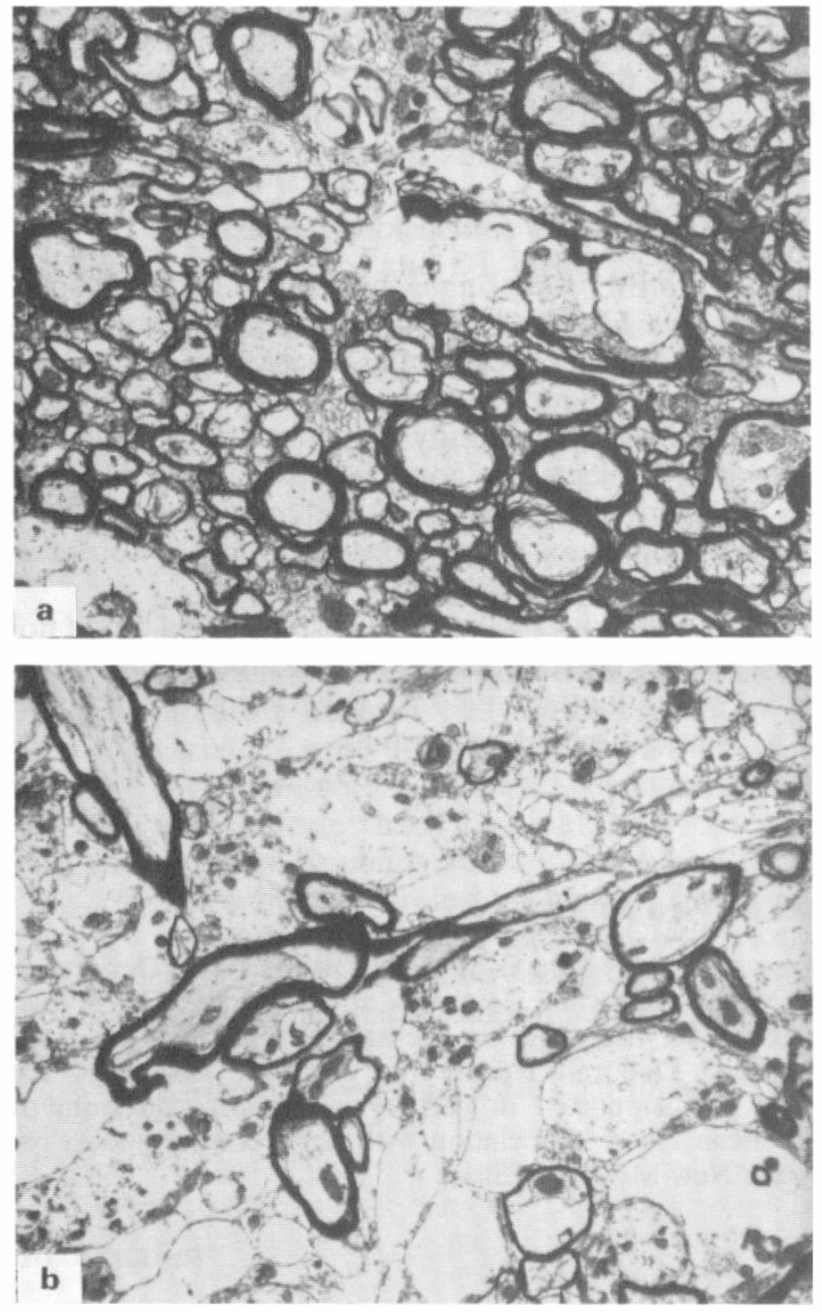

Figure 3 Ultrastructural appearance of lumbar spinal cord. (a) Normal rat with normal number, distribution and morphology of myelinated fibers. (b) Sections from spasticparaplegic rat show degeneration and loss of myelinated fibers, as well as vacuolation of the background neurophil. Uranyl acetate-lead citrate, $\times 6000$

area. In one animal, however, swelling and degeneration of axons was seen diffusely throughout the white matter of the posterior columns.

The region containing the corticospinal tract, which in the rat is in the dorsal columns, just dorsal to the gray matter, was studied ultrastructurally. Numerous degenerating axons with disintegrating myelin sheaths were seen (Figure $3 b$ ). Other axons had very thin myelin sheaths. In some axons, there appeared to be an empty space between the myelin sheath and the axoplasm. In many fibers, the axoplasm contained accumulations of microtubules, numerous dense bodies, myelin figures and degenerating organelles. Scavenger cells were present. The degeneration was very localized, and adjacent portions of the white matter appeared much better preserved. The location of these ultrastructural changes was confirmed by examining the grids under a light microscope; the area of observation was indeed in the area of the corticospinal tracts.
Spinal cord blood flow (SCBF) study

The data were analyzed with two-way ANOVA with level (cervical, thoracic, lumbar) and regions (medial dorsal column, ventral column, lateral column, lateral dorsal column, corticospinal tract, ventral horn) as factors. The test was followed by post-hoc multiple comparison (Fisher least significance difference method) among level and regions.

Analysis of variance showed a significant $P$ value both for level $(P=0.0001)$ and regions $(P=0.0001)$ but no interaction between the two $(P=0.94)$. Posthoc multiple comparison revealed that overall SCBF was not different in the lumbar and cervical cord, but it was significantly less in the thoracic cord. As expected, SCBF was higher in the gray (VH1) than in the white matter (MDC, VC, LC, LDC); interestingly, SCBF in the corticospinal tracts was significantly higher than the rest of the white matter and significantly lower than the gray matter (Figure 4 ).

\section{Discussion}

In our previous study of spinal cord ischemia secondary to aortic occlusion in the rat model, we characterized, on the basis of the histological and electrophysiological findings, two distinct presentations. In one group of animals the histological damage was confined to the white matter only. These animals did not have muscle degeneration, either histologically or by electromyographic recordings. The picture was compatible with an upper motor neuron lesion. In the second group, both gray and white matter were involved. Muscle atrophy was present and eletromyography (EMG) studies showed denervation. The picture was suggestive of combined upper and lower motor neuron lesions. Since spastic paraplegia was present also in this group, the upper motor neuron lesion was predominant. Interestingly, in both groups a lesion of the corticospinal tracts, known to descend in the most ventral portion of the

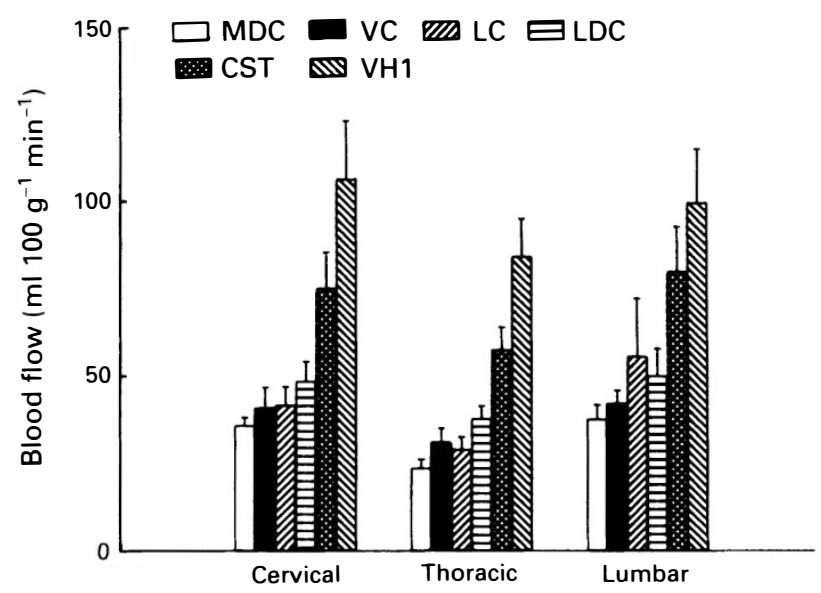

Figure 4 Spinal cord blood flow in six regions of cervical, thoracic and lumbar cord. Medial dorsal column (MDC), ventral column (VC), lateral column (LC), lateral dorsal column (LDC), corticospinal tract (CST) and ventral horn (VH1) 
dorsal columns in the rat ${ }^{5}$ was not seen. Likewise, all paraplegic animals demonstrated an increased SCCT, despite apparent sparing of the posterior tracts where the sensory evoked potentials are conducted. These data, however, were based on light microscopy of material embedded in paraffin, a technique which has significant limitations for this type of study.

Indeed, routine paraffin sections, while acceptable for evaluation of relatively severe spinal cord damage, are inadequate for the examination of nerve fiber morphology. This requires the use of resin-embedded sections employing osmium tetroxide post-fixation, which results in much-improved morphology of myelin sheaths with their lipid-rich composition. Resin processing is more laborious than paraffin processing, and requires smaller tissue sections, thus increasing the risk of sampling errors. Both time and sample size limitation are even more pronounced with electron microscopy. Inclusion of tissue from control animals which is handled in an identical manner to the experimental tissue is mandatory to avoid over-interpretation of artefacts that might be due to processing.

This investigation confirmed our previous findings of two types of lesions after spinal cord ischemia, the first characterized by white matter injury only and the second by involvement of both white and gray matters. In the group with spastic paraplegia, both light microscopy of resin-embedded sections and electron microscopy demonstrated that the nature of the upper motor neuron lesion is axonal degeneration, selectively localized to the corticospinal tract. Since active, on-going axonal degeneration was seen 32 days after the ischemic event, it may be true that a prolonged period of time may be required for the cellular damage to express itself morphologically. This degeneration, which is extremely focal in resin semi-thin sections, could not be identified in paraffin-embedded material.

Presumably, these considerations can be extended to the ascending tracts of the posterior columns, where electron microscopy studies were not carried out because of the expense and time required. Thus, we might speculate that similar ultrastructural changes, not visible on light microscopy, are present and are responsible for the abnormal transmission of the sensory evoked potentials and the increased SCCT. In this respect, the presence of diffuse axonal degeneration of posterior columns in the resin-embedded sections of one animal further supports this hypothesis.
The other significant finding of this study was that the blood flow of the corticospinal tract was considerably higher than the rest of the white matter regions. This observation may suggest a higher degree of metabolic activity in this tract which predisposes it to ischemia.

In summary a few conclusions can be made:

(1) Paraffin processing is inadequate for the study of nerve fiber morphology after spinal cord ischemia. Resin processing or electron microscopy are the techniques of choice.

(2) Ultrastructural study of the posterior columns of rats subjected to aortic occlusion showed axonal degeneration of the dorsal corticospinal tracts which may be responsible for the observed spastic paraplegia.

(3) In sham operated animals, spinal cord blood flow of the corticospinal tracts is highest among the white matter regions, suggesting an increased susceptibility to ischemia.

\section{Acknowledgements}

We would like to thank Clifford Qualls, PhD (Department of Mathematics and Statistics at the University of New Mexico) for assistance in the statistical evaluation of our data and Deborah Heuser for technical assistance. This study was supported in part by a Grant-in-Aid from the American Heart Association with funds contributed in part by the New Mexico Affiliate.

\section{References}

1 LeMay DR, Neal S, Zelenock GB, D'Alecy LG. Paraplegia in the rat induced by aortic cross-clamping: Model characterization and glucose exacerbation of neurologic deficit. J Vasc Surg 1987; 6: $383-390$

2 Follis $\mathrm{F}$ et al. Selective vulnerability of white matter during spinal cord ischemia. J Cereb Blood Flow Metab 1993; 13: 170-178.

3 Sakurada $\mathrm{O}$ et al. Measurement of local cerebral blood flow with iodo-[14C]-antipyrine. Am J Physiol 1978; 234: H59-H66.

4 Reivich M, Jehle J, Sokoloff L, Kety SS. Measurement of regional cerebral blood flow with antipyrine-[14C] in awake cats. J Appl Physiol 1969; 27: 296-300.

5 Paxinos G, Watson C. The Rat Brain in Stereotaxic Coordinates. Academic Press: New York. 